\title{
Percentual de suplementação de fonte taninífera na ração concentrada de caprinos jovens sobre o desempenho e carga parasitária
}

\author{
Tanniferous plant supplementation percentage in young goats \\ concentrates diet on perfermance and parasite control
}

\author{
Eduardo Shalders ${ }^{\mathrm{I}}$ Surama Freitas ZaniniI ${ }^{\mathrm{II}}$ Dyeime Ribeiro de Sousa ${ }^{\mathrm{I}}$ Graziela Barioni ${ }^{\mathrm{I}}$ \\ Renata Cogo Clipes ${ }^{\mathrm{III}}$ Barbara Rauta Avelar ${ }^{\mathrm{I}}$ Jamili Maria Mussi Suhet $^{\mathrm{IV}}$
}

\section{RESUMO}

O presente estudo buscou determinar o percentual de adição de torta de pimenta rosa (TPR) na dieta de caprinos jovens sobre o desempenho e carga parasitária. Foram utilizados 25 caprinos jovens da raça Saanen, distribuídos em um delineamento inteiramente casualizado em grupos de cinco tratamentos e cinco repetições, constituídos por grupos: não suplementados com TPR; suplementados com 15\% TPR; suplementados com 30\% TPR; suplementado com 45\% TPR; e suplementado com $60 \%$ TPR. O aumento dos níveis de suplementação de pimenta rosa resultou em efeito quadrático sobre o consumo voluntário de matéria seca (CVMS), peso metabólico e ganho em peso diário $(P \leq 0,05)$. Para o CVMS, o comportamento quadrático $(P \leq 0,05)$ revelou ponto de mínimo de 67,1 $\mathrm{kg}^{0,75} \mathrm{dia}^{-1}$ no percentual de 59,8\% de TPR; para o peso metabólico, um ponto máximo de $8,5 \mathrm{~kg}^{0,75}$ no percentual de 15,5\% de TPR; e, para o ganho em peso diário, um ponto máximo de 0,107g dia $\mathrm{d}^{-1}$ no percentual de 19,6\% de TPR na dieta. Em relação à influencia da TPR sobre a carga parasitária, observou-se efeito quadrático dos níveis de suplementação de TPR na contagem de ovos por grama de fezes $(O P G)(P \leq 0,05)$, com ponto de mínimo estimado em 1,0 $\log _{10}$ no nível de 35,8\% TPR na dieta, representando o limite de resposta do TPR. Além da redução no OPG, houve um decréscimo linear na contagem de oocistos por grama de fezes (OOPG) com o incremento nos níveis de TPR da dieta. Conclui-se que a adição de 20-30\% TPR possibilitou o controle de parasitos gastrointestinais com máximo desempenho animal.

Palavras-chave: taninos, Schinus terebinthifolius Raddi, desempenho, pequeno ruminante, oocisto.

\section{ABSTRACT}

The aim of this study was to evaluate the pink pepper supplementation (PPS) in young goats concentrate diet on performance parameters. Twenty five female Saanen goats were divided into five groups having five goats in each treatment. In a completely randomized design were given 0\%, 15\%, 30\%, 45\% and $60 \%$ of pink pepper supplementation in the diet. The increase levels of pink pepper supplementation resulted in a quadratic effect on voluntary intake of dry matter, metabolic weight and daily weight gain $(P \leq 0.05)$. For voluntary intake of dry matter, the quadratic effect $(P \leq 0.05)$ revealed minimum point of 67.1 ( $\mathrm{g} \mathrm{kg} \mathrm{g}^{0.75}$ $\mathrm{dia}^{-1}$ ) in the percentage of $59.8 \%$ PPS; for metabolic weight a peak of $8.5 \mathrm{~kg}^{0.75}$ in the percentage of $15.5 \%$ PPS, and the daily weight gain a peak of $0.107 \mathrm{~g} \mathrm{day}^{-1}$ in the percentage of $19.6 \%$ PPS in the diet. Regarding the effect of PPS on the parasite load, there was a quadratic effect of dietary supplementation of the PPS on egg count per gram of feces $(P \leq 0.05)$, with minimum point estimated at $1.0 \log _{10}$ at $35.8 \%$ of PPS in the diet, representing the limit of PPS response. There was a linear decrease on oocyst count per gram feces with increased levels of PPS 's diet. It was concluded that the supplementation up to 20-30\% of pink pepper was able to control of gastrointestinal parasites with maximum animal performance.

Key words: tannin, Schinus terebinthifolius Raddi, performance, small ruminant.

\section{INTRODUÇÃO}

A caprinocultura é amplamente difundida no mundo, com rebanho de aproximadamente 743,3 milhões de animais (FAO, 2009). No entanto, apresenta como entrave as parasitoses que, além de debilitar o animal, favorecem a instalação de outras patologias que comprometem a produção de leite e o rendimento de carcaça (PAIVA \& NEVES, 2009).

Muitos estudos têm utilizado plantas com atividade anti-helmíntica (NASCIMENTO et

\footnotetext{
'Programa de Pós-graduação em Ciências Veterinárias, Universidade Federal do Espírito Santo (UFES), Alegre, ES, Brasil.

IIDepartamento de Medicina Veterinária, UFES, 29500-000, Alegre, ES, Brasil. E-mail: smzanini@gmail.com. Autor para correspondência.

"IIInstituto Federal do Espírito Santo (IFES), Campus de Alegre, ES, Brasil.

${ }^{\text {IV }}$ Programa de Pós-graduação em Ciência Animal, Universidade Federal de Minas Gerais (UFMG), Belo Horizonte, MG, Brasil. Recebido 22.08.13 Aprovado 13.11.13 Devolvido pelo autor 12.04.14
} CR-2013-1131.R1 
al., 2009; SILVA et al., 2010; PARRA et al., 2011), pois essas plantas contêm compostos secundários, como taninos condensados (TC), que, além fornecer alternativas orgânicas para controle parasitário, não deixam resíduos em alimentos e diminuem custos de produção (ROEL, 2002; CHAGAS et al., 2004).

Embora a utilização de plantas, sementes, óleos ou extratos de vegetais seja comum no controle de parasitos em ruminantes, esta prática geralmente baseia-se em conhecimento empírico, sem comprovação da concentração recomendada (CABARET et al., 2002). Segundo GITHIORI et al. (2006), os testes in vitro são mais realizados para determinar a ação anti-helmíntica das plantas, pois têm as vantagens de ser de baixo custo, rápido resultado, permite a triagem de uma grande quantidade de plantas e a possibilidade de avaliar substâncias isoladas sem interferência de outros compostos. Contudo, estes testes avaliam o efeito do TC sobre os nematoides sem a interferência fisiológica do animal, ou seja, não se observam os possíveis efeitos antinutricionais dos taninos no desempenho (KETZIS et al., 2006; CABARET et al., 2002).

Segundo OLIVEIRA et al. (2011), a presença de plantas taniníferas pode influenciar positivamente na sanidade de pequenos ruminantes, entretanto, seus efeitos dependem do tipo e da concentração desses metabólitos. Dessa forma, não existe uma indicação precisa de como utilizá-los no controle de nematoides gastrintestinais de pequenos ruminantes, por isso há necessidade de mais estudos. Entretanto, HOSTE et al. (2006) relatam que o consumo de altas concentrações de $\mathrm{TC}$, maior que $7 \%$ da matéria seca, reduz a ingestão alimentar, inibe o crescimento animal, interfere na morfologia e na atividade proteolítica de microrganismos ruminais, todavia, concentrações baixas ou moderadas de TC, menor que $6 \%$ da matéria seca, promove aumento na produção e diminuem o parasitismo intestinal.

Schinus terebenthifolius Raddi, também chamada de pimenta rosa, é uma espécie nativa pertencente à família Anacardiaceae, que contém flavonoides, triterpenos, saponinas e fenóis como taninos (LIMA et al., 2006), além disso, apresenta potencial de utilização na alimentação animal (GONÇALVES et al., 2012). Por isso, este estudo buscou determinar o percentual de inclusão de torta de pimenta rosa (TPR) na dieta caprina de animais jovens, sem prejudicar o desempenho animal, mas que possa atuar como controle parasitário.

\section{MATERIAL E MÉTODOS}

O experimento foi realizado nas instalações para caprinos do Rancho Visoleta Capril $\mathrm{SH}$, localizado no município de Itapemirim-ES, pelas coordenadas geográficas $20^{\circ} 53{ }^{\prime} 51^{\prime}$ S, longitude $40^{\circ} 52^{\prime} 38^{\prime \prime} \mathrm{W}$, apresentando uma altitude de $9 \mathrm{~m}$. O clima da região é quente e úmido (tropical), segundo a classificação de KÖPPEN-GEIGER, descrita por KOTTEK et al. (2006).

Foram utilizados 25 caprinos jovens da raça Saanen, clinicamente saudáveis, que foram pesados, identificados individualmente por brincos plásticos numerados e afixados nas orelhas, com idade entre 50 a 70 dias e com peso vivo médio de $12 \mathrm{~kg}$, distribuídos em delineamento inteiramente casualizado, em grupos de cinco tratamentos e cinco repetições.

Os tratamentos foram constituídos pelos grupos: não suplementados com TPR (NS), suplementados com 15\% TPR (S15), suplementados com 30\% TPR (S30), suplementados com $45 \%$ TPR (S45) e suplementados com 60\% TPR (S60). A pimenta rosa substituiu até $60 \%$ do milho. As dietas foram formuladas para atender aos requerimentos de ganhos médios diários de 100g/animal/dia, segundo o NATIONAL RESEARCH COUNCIL (2007).

A torta de pimenta rosa apresentou os valores ( $\%$ na MS) de $7 \%$ de proteína bruta, $10 \%$ de extrato etéreo, $21 \%$ de fibra bruta, $45 \%$ de fibra detergente neutro e $23 \%$ de fibra detergente ácido, estimados de acordo com a metodologia estabelecida por SILVA (1998). Os valores de fenóis totais, de taninos totais e de taninos condensados foram de 2,86 em equivalente grama de ácido tânico por $100 \mathrm{~g}$ de matéria seca (MS), de 2,49 em equivalente grama de ácido tânico por $100 \mathrm{~g}$ de MS e de 0,18 , em equivalente grama de leucocianidina por $100 \mathrm{~g}$ de MS, respectivamente. O conteúdo dos compostos fenólicos foram determinados com base nos trabalhos de PORTER et al. (1986) e MAKKAR et al. (1993).

Os animais foram mantidos em regime de confinamento, em baias individuais, medindo 0,50x1,2m, em galpão coberto, com piso ripado, providas de comedouro e bebedouro, recebendo água à vontade e alimento volumoso duas vezes ao dia, totalizando, em média, 4,0\% do peso vivo (PV), em quantidade suficiente para permitir uma sobra entre 10 e $15 \%$ do oferecido. Inicialmente, os animais foram submetidos a um período de 14 dias de adaptação às instalações, ao manejo, à dieta e ao ajuste do consumo animal. O período experimental teve duração de sete semanas, sendo que não houve tratamento antihelmíntico, antes ou durante o período experimental. 
Os parâmetros de desempenho avaliados foram consumo voluntário de matéria seca (CVMS), como a diferença entre as quantidades de matéria seca oferecida e refugada, expresso em gramas por unidade de peso metabólico $\left(\mathrm{g} \mathrm{kg}^{0,75} \mathrm{dia}^{-1}\right)$; peso metabólico $\left(\mathrm{kg}^{0,75}\right)$; ganho em peso diário $\left(\mathrm{g} \mathrm{dia}^{-1}\right)$, em que a pesagem individual dos animais foi executada em intervalos de sete dias até o fim do experimento, mediante jejum.

Para avaliação parasitológica, as fezes foram coletadas durante o período experimental, em intervalos de sete dias, direto da ampola retal em sacos plásticos de $03 \times 12 \mathrm{~cm}$, devidamente lubrificados com glicerina. As amostras foram identificadas, acondicionadas em caixas de isopor com gelo e encaminhadas ao laboratório de Doenças Parasitarias da Universidade Federal do Espírito Santo (UFES) para o processamento. Os exames foram realizados de acordo com as técnicas descritas por GORDON \& WHITLOCK (1939). Os resultados da contagem de ovos (OPG) e de oocistos (OOPG) por grama de fezes foram transformados para $\log _{10}(\mathrm{x}+10)$, visando à uniformização da variância das médias.

Os resultados dos parâmetros avaliados foram submetidos à análise de variância (ANOVA), de regressão, em função dos tratamentos, e de correlação de Pearson, utilizando o pacote computacional SAEG da Universidade Federal de Viçosa (UFV) (1997), considerando o nível de significância de $5 \%(\mathrm{P}<0,05)$. Os efeitos dos níveis de TPR foram estimados por meio de modelos de regressão linear e quadrática.

\section{RESULTADOS E DISCUSSÃO}

O desempenho animal foi significativamente influenciado pelo nível de suplementação de pimenta rosa na dieta $(\mathrm{P} \leq 0,05)$. $\mathrm{O}$ aumento dos níveis de suplementação de pimenta rosa resultou em efeito quadrático sobre o consumo voluntário de matéria seca, peso metabólico e ganho em peso diário $(\mathrm{P} \leq 0,05)$. Para o CVMS, o comportamento quadrático $(\mathrm{P} \leq 0,05)$ revelou ponto de mínimo de $67,1 \mathrm{~g} \mathrm{~kg}^{-0,75} /$ dia no percentual de 59,8\% de TPR (Figura 1A), para o peso metabólico um ponto máximo de $8,5 \mathrm{~kg}^{0,75}$ no percentual de $15,5 \%$ de TPR (Figura 1B), e, para o ganho em peso diário, um ponto máximo de $0,107 \mathrm{~g}$ dia $^{-1}$ no percentual de $19,6 \%$ de TPR na dieta (Figura 1C). Sendo assim, a suplementação de 15-20\% TPR maximizou a performance, embora o CVMS tenha sido reduzido (Figura 1).

A alteração no CVMS (g kg-0,75 dia $\left.^{-1}\right)$ no peso metabólico $\left(\mathrm{g} \mathrm{kg}^{-0,75}\right)$ e no ganho em peso diário (g/dia) relacionados à suplementação com TPR podem ter sido influenciados pelas concentrações TC na dieta, devido à sensação de adstringência provocada pela formação dos complexos entre os taninos e as glicoproteínas salivares (REED, 1995), que diminui a palatabilidade e, por consequência, o consumo (OTERO \& HIDALGO, 2004). Apesar disso, em ruminantes, os taninos podem promover efeitos positivos, como reduzir a quantidade de proteína digerida no rúmen e aumentar a quantidade de proteína disponível no intestino delgado, eliminar parasitas e diminuir o timpanismo espumoso (MUELLER-HARVEY, 2010).

Os dados do presente estudo assemelhamse aos observados por GOMES et al. (2010), que relataram redução no consumo e no ganho de peso em cordeiros alimentados com resíduo agroindustrial da carnaúba, devido à baixa digestibilidade e à alta concentração de taninos (12,2\% de matéria seca). Segundo LEMPP (2007) e MIN et al. (2003), o alto teor de tanino pode interferir diretamente na digestibilidade de matéria seca, matéria orgânica, proteínas, carboidratos e fibras, afetando negativamente a produtividade.

Em relação à influencia da TPR sobre a carga parasitária, observou-se efeito quadrático dos níveis de suplementação de TPR no $\mathrm{OPG}(\mathrm{P} \leq 0,05)$, com ponto de mínimo estimado em $1,0 \log _{10}$ (Figura 2A) no nível de 35,8\% TPR na dieta, representando o limite de resposta do TPR. Acredita-se que a diminuição do OPG em animais tratados com TPR, seja resultado dos TC atuando na eliminação dos vermes, ou redução da fecundidade das fêmeas. Tais efeitos já foram relatados em pequenos ruminantes, suplementados com fontes vegetais ricas em taninos, por MUPEYO et al. (2011), JOSHI et al. (2011), PARRA et al. (2011) e NOGUEIRA et al. (2012).

SILVA et al. (2010) também observaram uma redução no número médio de OPG em caprinos naturalmente infectados por nematoides alimentados com Typha domingensis (taboa) e da Operculina hamiltonii (batata de purga). Entretanto, NASCIMENTO et al. (2009) avaliaram o efeito de frutos do umbuzeiro in natura sob o ganho de peso e efeito anti-helmíntico em caprinos e verificaram que não houve diferença significativa $(\mathrm{P}>0,05) \mathrm{em}$ nenhum dos parâmetros avaliados.

A redução do OPG pode diminuir a contaminação da pastagem e a dinâmica da infecção no animal (HECKENDORN et al., 2007). Os TC também podem atuar sobre a eclosão e o desenvolvimento das larvas dos nematoides, pois, como não são absorvidos pelo trato gastrintestinal dos pequenos ruminantes, ocorre sua eliminação nas fezes e redução da contaminação do 


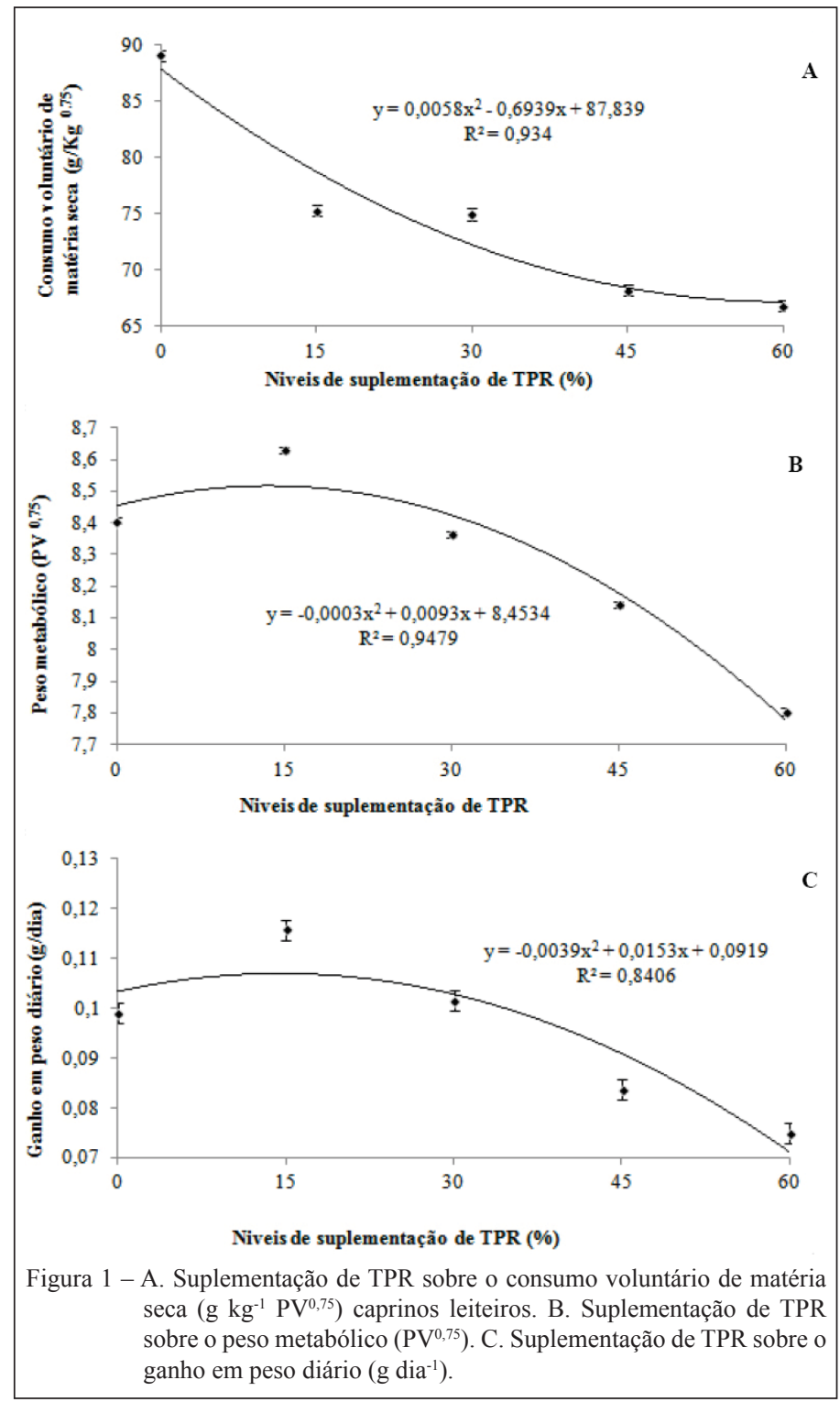

pasto (MUPEYO et al., 2011). Experimentos in vivo indicaram que o consumo de plantas taniníferas também diminuiu significativamente o estabelecimento de L3 (JOSHI et al., 2011).

Este estudo revelou que, além da redução no OPG, houve uma redução linear na contagem de oocistos por grama de fezes (OOPG) com o incremento nos níveis de TPR da dieta (Figura 2B). Através da análise de correlação simples, foi verificada correlação negativa entre a adição de níveis crescentes da TPR e a contagem total de OOPG durante o período experimental (Figura 2B).

HUR et al. (2005) observaram uma diminuição acentuada na produção de oocistos em cabras, alimentadas com Pinus densifora, Quercus acutissima, plantas ricas em TC, essa queda na produção de oocistos pode ser a ação direta dos TC em diferentes fases do ciclo de vida do parasita, ao invés de através de uma melhor nutrição proteica das cabras.

\section{CONCLUSÃO}

Concluiu-se que a adição de $20-30 \%$ de torta de pimenta rosa possibilitou controle de parasitos gastrointestinais com máximo desempenho animal.

\section{AGRADECIMENTOS}

Ao Centro Nacional de Pesquisa e Desenvolvimento $(\mathrm{CNPq})$, pelo apoio financeiro concedido para execução desta pesquisa. 


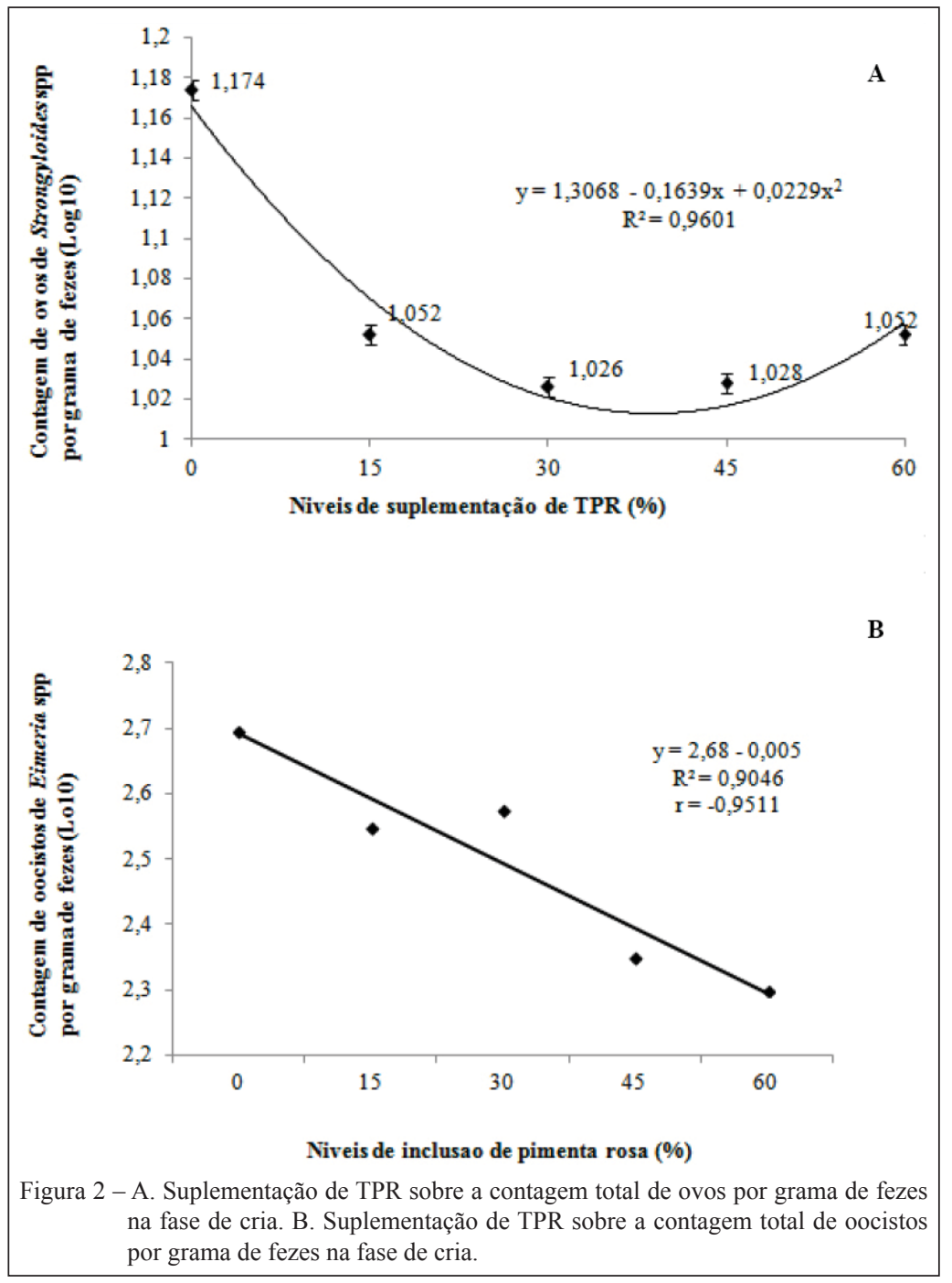

\section{COMITÊ DE ÉTICA}

O protocolo de experimentação animal está de acordo com Congresso Nacional dos Estudantes de Agronomia (CONEA) e foi aprovado pelo comitê de ética no uso de animais (CEUA) da Universidade Federal do Espírito Santo (UFES) pelo protocolo n.34/13.

\section{REFERÊNCIAS}

CABARET, J. et al. Managing helminths of ruminants in organic farming. Veterinary Research, v.33, n.5, p.625-640, 2002.

CHAGAS, A.C.S. et al. Controle de parasitas utilizando extratos vegetais. Revista Brasileira de Parasitologia Veterinária, v.13, supl.1, p.156-160, 2004.

FAO Statistical Database - FAOSTAT. Disponível em: <http:/ faostat.fao.org/>. Acesso em: 21 dez. 2009 e 15 ago. 2011.

GITHIORI, J.B. et al. Use of plants in novel approaches for control of gastrointestinal helminths in livestock with emphasis on small ruminants. Veterinary Parasitology, v.139, n.4, p.308-320, 2006.
Disponível em: <http://www.sciencedirect.com/science/article/pii/ S0304401706002548>. Acesso em: 28 novembro de 2012.

GOMES, R.V.R.S. et al. Ação antiparasitária in vitro dos extratos etanólicos de Operculina hamiltonii (batata de purga) e Momordica charantia (melão de são caetano) sobre ovos e larvas de nematóides gastrintestinais de caprinos do semi-árido paraibano. Acta Veterinaria Brasilica, v.4, n.2, p.92-99, 2010

GONÇALVES, F.G. et al. Efeito da pimenta rosa associada a diversas dosagens de antibióticos em frangos de corte. Ciência Rural, v.42, n.8, p.1503-1509, 2012. Disponível em: <http:// dx.doi.org/10.1590/S0103-84782012005000055>. Acesso em: 12 outubro de 2012.

GORDON, H.M.; WHITLOCK, H.V. A new technique for courting nematode eggs in sheep faeces. Journal of Council of Science and Industry Research in Australia, v.12, p.50-52, 1939.

HECKENDORN, F. et al. Individual administration of three tanniferous forage plants to lambs artificially infected with Haemonchus contortus and Cooperia curticei. Veterinary Parasitology, v.146, n.1-2, p.123-134, 2007. 
HOSTE, H. et al. The effects of tannin-rich plants on parasitic nematodes in ruminants. Trends in Parasitology, v.22, n.6, p.253-261, 2006. Disponível em: <http://www.sciencedirect. com/science/article/pii/S1471492206000997>. Acesso em: 11 outubro de 2012 .

HUR, S.N. et al. Effects of feeding condensed tannin-containing plants on natural coccidian infection in goats. Asian-Australasian Journal of Animal Sciences, v.18, n.9, p.1262-1266, 2005.

JOSHI, B.R. et al. Effect of feeding sericea lespedeza leaf meal in goats experimentally infected with Haemonchus contortus Veterinary Parasitology, v.178,n.1-2,p.192-197,2011. Disponível em: <http://www.ncbi.nlm.nih.gov/pubmed/21232867>. Acesso em: 10 de outubro de 2012

KETZIS, J.K. et al. Evaluation of efficacy expectations for novel and non-chemical helminth control strategies in ruminants. Veterinary Parasitology, v.139, n.4, p.321-335, 2006.

KOTTEK, M. et al. World map of the Köppen-Geiger climate classification updated. Meteorologische Zeitschrift, v.15, p.259263,2006

LEMPP, B. Avanços metodológicos da microscopia na avaliação de alimentos. Revista da Sociedade Brasileira de Zootecnia, v.36, p.315-329, 2007.

LIMA, M.R.F. et al. The antibiotic activity of some Brazilian medicinal plants. Revista Brasileira de Farmacognosia, v.16, n.3, p.300-306, 2006.

MAKKAR, H.P.S. et al. Gravimetric determination of tannins and their correlation with chemical and protein precipitation methods. Journal of the Science of Food and Agriculture, v.61, n.2, p.161-165, 1993. Disponível em: <http://onlinelibrary.wiley.com/ doi/10.1002/jsfa.2740610205>. Acesso em: 09 de outubro de 2012.

MIN, B. R. et al. Direct effects of condensed tannins on gastrointestinal nematodes in grazing Angora goats. Journal of Animal Science, v.81, n.2, p.23, 2003.

MUELLER-HARVEY, I. Unravelling the conundrum of tannins in animal nutrition and health. Journal of the Science of Food and Agriculture, v.86, n.13, p.1097-0010, 2010. Disponível em: $<$ http://dx.doi.org/10.1002/jsfa.2577>. Acesso em: 09 ago. 2011.

MUPEYO, B. et al. Effects of feeding willow (Salix spp.) upon death of established parasites and parasite fecundity. Animal Feed Science and Technology, v.164, n. 1-2, p.8-20, 2011 Disponível em: <http://www.sciencedirect.com/science/article/pii/ S0377840110003974>. Acesso em: 30 setembro de 2012

NASCIMENTO, T.V.C et al. Desempenho produtivo e controle de nematódeos gastrintestinais em caprinos suplementados com frutos do umbuzeiro na Região Semiárida. Revista Brasileira de Agroecologia, v.4, n.2, p.2855-2858, 2009.
NATIONAL RESEARCH COUNCIL - NRC. Nutrient Requirements of Small Ruminants: Sheep, Goats, Cervids, and New World Camelids. 1.ed. Washington, D.C.: National Academy Press, 2007. 384p

NOGUEIRA, F.A. et al. Anthelminthic efficacy of banana crop residues on gastrointestinal nematodes of sheep: in vitro and in vivo tests. Parasitology Research, v. 111, n. 1, p. 317-323, 2012.

OLIVEIRA, L.M.B. de et al. Plantas taniníferas e o controle de nematóides gastrintestinais de pequenos ruminantes. Ciência Rural, v.41, n.11, p. 1967-1974, 2011. Disponível em: <http:// www.scielo.br/scielo.php?script $=$ sci arttext\&pid $=\mathrm{S} 01038478$ 2011001100019\&ng=en\&nrm=iso $>$. Acesso em: 20 out. 2012. Doi:10.1590/S010384782011001100019.

OTERO, M.J.; HIDALGO, L.G. Taninos condensados en espécies forrajeras de clima templado: efectos sobre productividad de rumiantes afectados por parasitosis gastrointestinales. Livestock Research for Rural Development, v.16, n.2, p.1-9, 2004.

PAIVA; L.J.M.; NEVES, M.F. Controle orgânico de parasitas Revista Cientifica Eletrônica de Medicina Veterinária, Ano VII, v.12, 2009 .

PARRA, C.L.C. et al. Alteração da carga de endoparasitas em ovinos submetidos a diferentes níveis de folha de bananeira na alimentação. Revista Brasileira de Agroecologia, v.6, n.2, p.111-116, 2011

PORTER, L.J. et al. The conversion of procyanidins and prodelphinidins to cyanidin and delphinidin. Phytochemistry, v.25, n.1, p.223-230, 1986.

REED, J.D. Nutritional toxicology of tannins and related polyphenols in forage legumes. Journal of Animal Science, v.73, n.5, p.1516-1528, 1995

ROEL, A.R. Utilização de plantas com propriedades inseticidas: uma contribuição para o Desenvolvimento Rural Sustentável. Revista Internacional de Desenvolvimento Local, v.1, n.2, p.43-50, 2002

SILVA, C.F. et al. Avaliação da eficácia de taboa (Typha domingensis Pers.) e batata-de-purga Operculina hamiltonii (G. Don) D.F. Austin \& Staples in natura sobre nematóides gastrintestinais de caprinos, naturalmente infectados, em clima semi-árido. Revista Brasileira de Plantas Medicinais, v.12, n.4, p. 466-471, 2010. Disponível em: <http://www.scielo.br/scielo. php?script=sci_arttext\&pid=S151605722010000400010\&lng= en\&nrm=iso $>$. Acesso em: 25 out. 2012. doi: 10.1590/S151605722010000400010

SILVA, D.J. Análise de alimentos (métodos químicos e biológicos). 2.ed. Viçosa: UFV, 1998. 165p.

UNIVERSIDADE FEDERAL DE VIÇOSA (UFV). Manual de utilização do programa SAEG. Viçosa, MG: UFV, 1997. 150p. 